\title{
Supramolecular Polymerization Provides Non-Equilibrium Product Distributions of Imine-Linked Macrocycles
}

\author{
Michael J. Strauss, Austin M. Evans, Ioannina Castano, Rebecca L. Li, and William R. Dichtel* \\ Department of Chemistry, Northwestern University, Evanston, IL, 60208 United States
}

\begin{abstract}
Supramolecular polymerization of imine-linked macrocycles has been coupled to dynamic imine bond exchange within a series of macrocycles and oligomers. In this way, macrocycle synthesis is driven by supramolecular assembly, either into small aggregates supported by $\pi-\pi$ interactions, or high-aspect ratio nanotubes stabilized primarily by electrostatic and solvophobic interactions. For the latter, supramolecular polymerization into nanotubes restricts imine exchange, thereby conferring chemical stability to the assemblies and their constituent macrocycles. Competition in the formation and component exchange among macrocycles favored pyridine-2,6-diimine-linked species due to their rapid synthesis, thermodynamic stability, and assembly into high-aspect ratio nanotubes under the reaction conditions. In addition, the pyridine-containing nanotubes inhibit the formation of similar macrocycles containing benzene-1,3-diimine-linkages, presumably by disrupting their assembly and templation. Finally, we exploit rapid imine exchange within weak, low-aspect ratio macrocycle aggregates to carry out monomer exchange reactions to macrocycles bearing pyridine moieties. Once a pyridine-containing dialdehyde has exchanged into a macrocycle, the macrocycle becomes capable of nanotube formation, which dramatically slows further imine exchange. This kinetic trap provides chemically diverse macrocycles that are not attainable by direct synthetic methods. Together these findings provide new insights into coupling supramolecular polymerization and dynamic covalent bond-forming processes, and leverages this opportunity to target asymmetric nanotubes. We envision these findings spurring further research efforts in the synthesis of nanostructures with designed and emergent properties.
\end{abstract}

\section{Introduction}

Supramolecular polymers are a compelling platform to design nanostructures with diverse functionality, long range order, and dynamic properties that are not attainable via traditional covalent polymerization. ${ }^{1-5}$ Due to the promise of accessing materials with these sought-after properties, the last decade of research has seen an emergence in novel supramolecular polymerization strategies such as 'sergeant and soldier' chirality amplification, ${ }^{6-9}$ living supramolecular polymerization, ${ }^{10-14}$ and supramolecular (co)polymerization. ${ }^{5,15-18}$ While these strategies allow access to diverse nanostructures, they all employ a general two-step process in which the building blocks are first isolated as a unimolecular species and polymerized in a second synthetic step. In this process, polymerization is typically induced by altering the solvent composition ${ }^{19-23}$ or changing the temperature of a monomer solution. ${ }^{5,24-27}$ Using this approach, the chemical structure of the building blocks remain fixed and diverse nanostructures emerge from the order in which they assemble. However, supramolecular polymerization under conditions in which the monomers can also undergo structural changes can target highly diverse nanostructures that are kinetically stabilized by the function of supramolecular polymerization. ${ }^{28-30}$

Using the traditional two-step approach, we have previously isolated imine-linked macrocycles derived from aromatic dialdehydes and a bifunctional aryl amine (DAPB); and studied their aptitude to undergo acid-mediated supramolecular polymerization into high-aspect ratio nanotubes.31-32 In the case of macrocycles derived from simple aromatic dialdehydes such as terephthaldehyde and isophthalaldehyde 
(IDA, MC 1), high concentrations of $\mathrm{CF}_{3} \mathrm{CO}_{2} \mathrm{H}$ (>2000 equiv) were required to protonate the imine linkages and drive assembly, while lower acid concentrations catalyzed macrocycle hydrolysis. ${ }^{31-32}$ However, including pyridine moieties (MC 2), which are significantly more basic than the imine linkages allowed macrocycle assembly to occur upon pyridinium formation, even in the presence of sub-stoichiometric acid loadings. ${ }^{31}$ Because of the low concentrations of $\mathrm{CF}_{3} \mathrm{CO}_{2} \mathrm{H}$ needed for supramolecular polymerization, we hypothesized that pyridine-containing macrocycles would form nanotubes during their covalent synthesis. In this way, the process of supramolecular polymerization and imine-exchange amongst pyridine containing species may interact and influence each other. Furthermore, we hypothesized that the relative strengths of non-covalent interactions supporting the assemblies of MC 1 and MC 2 would have profound effects on their kinetic stability and ability to undergo monomer exchange. Under this hypothesis, the relatively weak $\pi-\pi$ interactions supporting $\mathbf{M C} \mathbf{1}$, coupled with the inherent reactivity of IDA and 2,6-pyridinedicarboxaldehyde (DFP), would enable monomer exchange to macrocycles bearing pyridine moieties. This exchange would unlock nanotube formation and provide non-symmetric macrocycles that are inaccessible by direct synthetic methods (Figure 1).

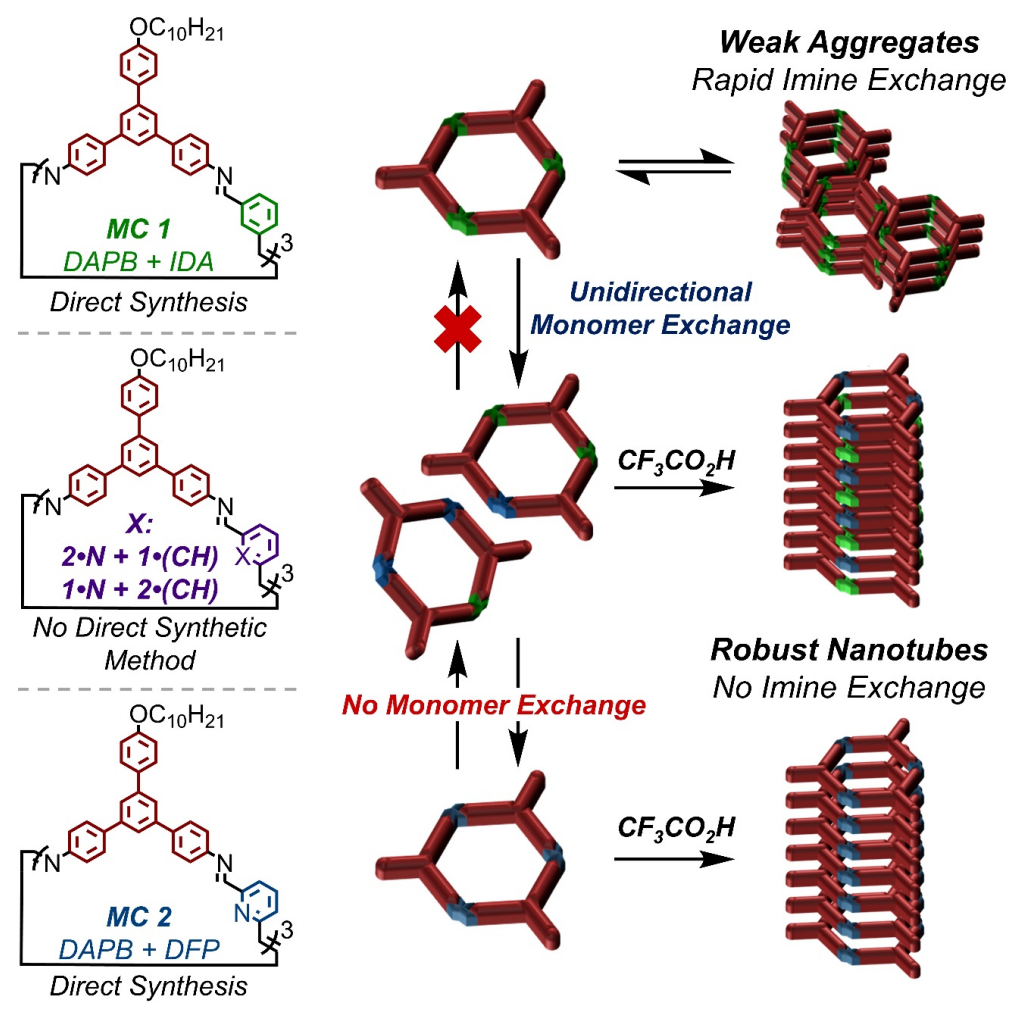

Figure 1. Impacts of pyridine moiety incorporation on macrocycles aptitude to undergo acid-mediated supramolecular polymerization, and the impacts of supramolecular polymerization on imine dynamics. 


\section{Results and Discussion}

The reactivity of DFP along with the assembly of MC 2 into nanotubes under reaction relevant conditions accelerates the formation of MC 2 relative to MC 1 by at least two orders of magnitude (Figure 2A). The assembly processes of nanotube formation of charged macrocycles and $\pi-\pi$ aggregation of neutral macrocycles that each drive macrocyclization cause the relative rates of macrocycle formation to be correlated to the emergence of an X-ray diffraction (XRD) signal. Tracing the emergence of the predominant nanotube diffraction feature via time-resolved XRD (TR-XRD) demonstrates that the formation of MC 2 occurs before the first data point was obtained $(2.5 \mathrm{~min}$ ), while the formation of $\mathbf{M C} \mathbf{1}$ takes over two hours (Figure 2B and 2E). The results of TR-XRD were validated using gel-permeation chromatography (GPC), in which MC 2 demonstrated a single narrow elution band after 2 minutes of reaction time. However, in the case of MC 1,2 minutes of reaction time yielded linear polymer and as the major product, with a small
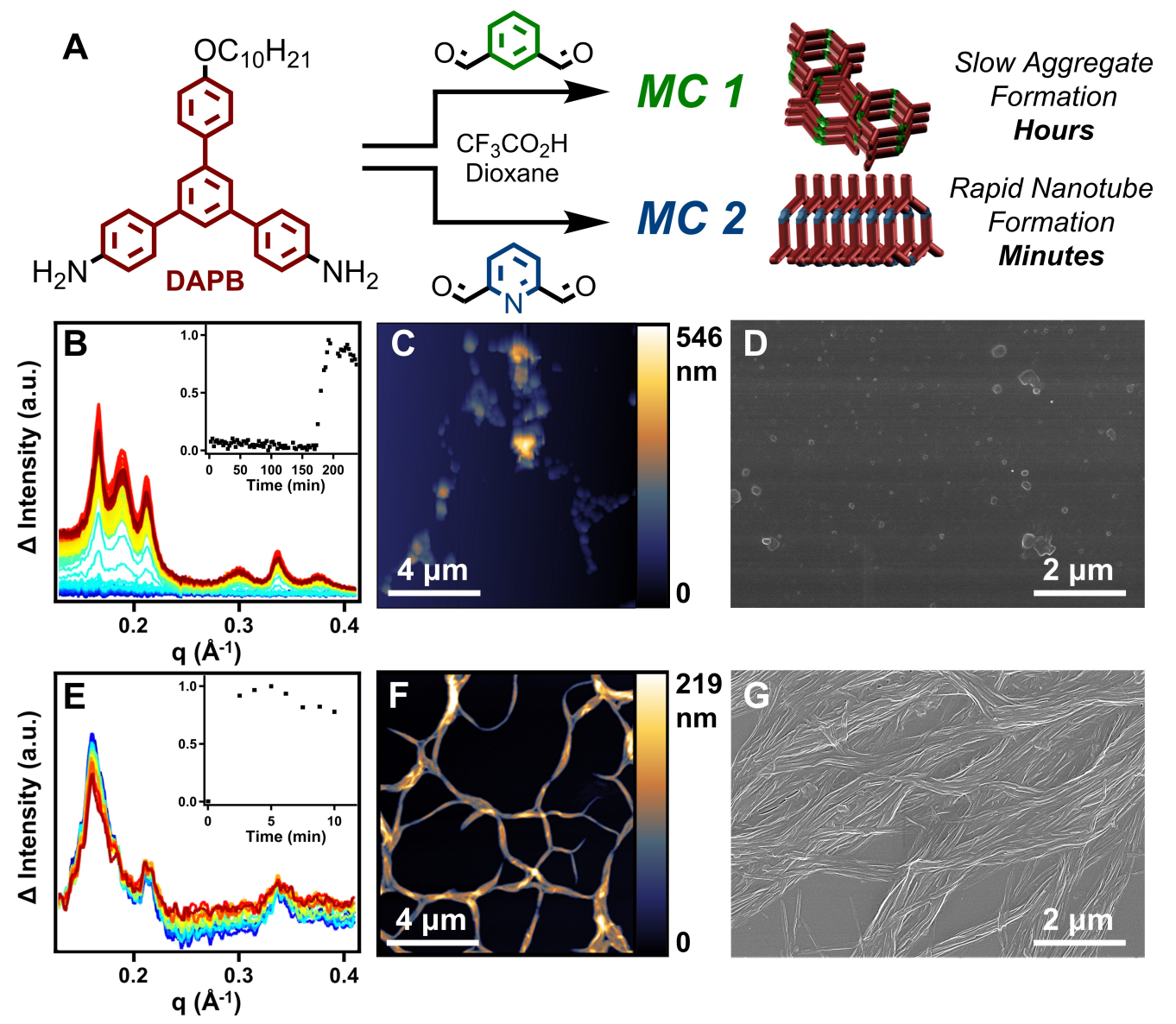

Figure 2. Probing the kinetics of the assembly processes that govern the formation of MC $\mathbf{1}$ and MC 2. (A) Scheme of macrocycle formation. (B) TR-XRD patterns from 0 to 240 minutes depicting the formation and assembly of MC 1. (Inset) Normalized integration of the diffraction signal with respect to time. (C) Atomic force micrograph of the ill-defined aggregates resulting from the formation of $\mathbf{M C}$ 1. (D) Scanning electron micrograph of the ill-defined aggregates resulting from the formation of MC 1. (E) TR-XRD patterns from 0 to 10 minutes depicting the rapid formation and assembly of MC 2 . Slight decreases in intensity were observed due to X-ray beam damage of the resulting nanotubes. (Inset) Normalized integrations of the diffraction signal with respect to time. (F) Atomic force micrograph of the nanotubes resulting from the formation of MC 2. (G) Scanning electron micrograph of the nanotubes resulting from the formation of MC 2. 
secondary peak corresponding to the target macrocycle. At longer times, the GPC signal of the MC 1 experiment narrowed, indicating successful macrocycle formation (See Supporting Information), which is consistent with our previous study on how neutral macrocycles form via linear polyimines. ${ }^{33}$ Characterization of the resulting macrocycles by matrix-assisted laser desorption ionization mass spectrometry (MALDI-MS) revealed single peaks corresponding to the target macrocycles (Figures S4 and S23). Furthermore, analysis of the final states of each macrocyclization reaction by atomic force microscopy (AFM), scanning electron microscopy (SEM), and transmission electron microscopy (TEM) demonstrated that MC 2 forms high aspect ratio nanotubes, while MC 1 yield ill-defined aggregates, which is consistent with previous reports (Figures $2 \mathrm{C}-2 \mathrm{D}$ and $2 \mathrm{~F}-2 \mathrm{G}$ ) (See Supporting Information). ${ }^{31,33}$ Contributing factors to the observed rate acceleration are the inductive nature of the pyridine ring, which increase the rate of imine condensation to yield acyclic products, and supramolecular polymerization, which drives imine-exchange of these undesired intermediates to yield MC 2 ${ }^{34-37} \mathrm{~A}$ small molecule study in which DFP was condensed with aniline under conditions typical for macrocycle synthesis yielded 2,6diiminophenylpyridine within the first five minutes of reaction time, which is an order of magnitude faster than the reaction of aniline with IDA (Figure S110-114). The above experiments demonstrate that MC 2 assembles into nanotubes under conditions typical for its synthesis and that its linkages form more rapidly than those of MC 1. These two factors explain the rapid and highly selective formation of MC 2, and we designed follow-up experiments to further explore this interplay.

The formation of MC 2 dominates a competition experiment in which both dialdehydes compete for a limited number of amine nucleophiles (Figure 3A). By combining 1 equiv of DAPB with 1 equiv each of DFP and IDA MC 2 is formed in high yield while reaction of IDA with small quantities of DAPB yields acyclic oligomers. In order to ensure that the formation of a small population of MC 1 was not limited by

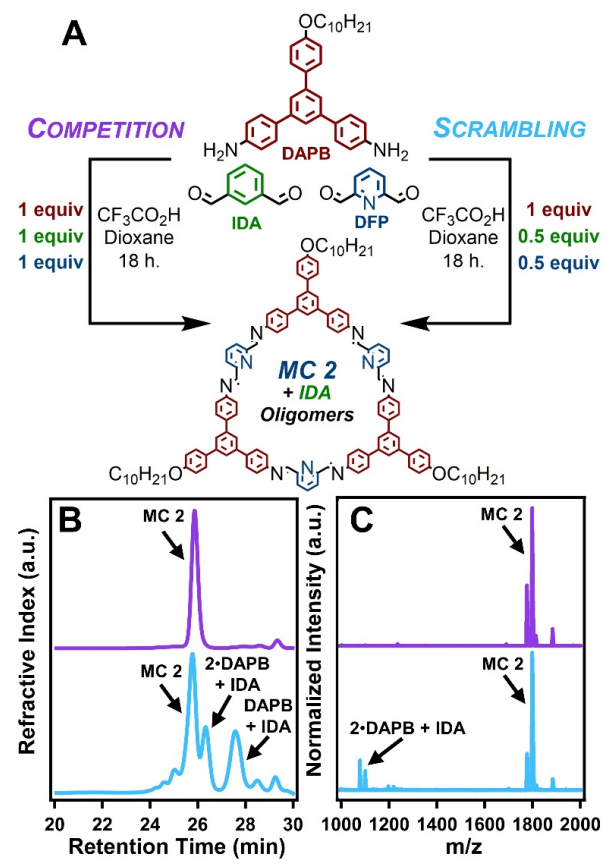

Figure 3. Probing the interplay of kinetic preference and chemical stability of MC 2 through competition and scrambling experiments. (A) Scheme of the competition and scrambling experiments. (B) Representative gel permeation chromatograms of the competition experiment (purple) and the scrambling experiment (blue). (C) Representative MALDI-MS spectra of the results of the competition experiment (purple) and the scrambling experiment (blue). MC 2 was observed as the $[\mathrm{M}+\mathrm{H}]^{+}$, $[\mathrm{M}+\mathrm{Na}]^{+}$, and $[\mathrm{M}+\mathrm{K}]^{+}$adducts. 
availability of the acid catalyst, the reaction was run at an elevated acid loading (10 equiv). The products of this competition reaction were characterized by GPC, MALDI-MS, and ${ }^{1} \mathrm{H}$ NMR spectroscopy. GPC analysis indicated the predominant formation of a single macrocyclic product, as judged by the narrow peak shape whose retention time matched purified samples of MC 2, along with relatively weak signals corresponding to other oligomeric species (Figure 3B, purple trace). MALDI-MS analysis of the same product mixture indicated a strong signal corresponding to the mass of MC 2, along with small oligomers containing IDA and DAPB species that did not react to form macrocycle (Figures 3C and S78-S82). No signal corresponding to MC 1 was observed. ${ }^{1} \mathrm{H}$ NMR spectroscopy of the crude products also indicated the selective formation of MC 2. Similar to the MALDI-MS analysis, no signals in the NMR spectrum corresponding to MC 1 were observed (Figure S83). The formation of nanotubes under the reaction conditions was confirmed by AFM, SEM, and TEM; demonstrating that small oligomers containing IDA moieties did not interrupt the assembly of MC 2 (Figures S84-S86). Lastly, the presence of assembled nanotubes in the reaction solution prior to workup was confirmed by in situ XRD, which yielded a pattern comparable to the direct synthesis of MC 2 (Figure S87). Finally, a small molecule competition study in which IDA (1 equiv), DFP (1 equiv), and aniline ( 2 equiv) were reacted under conditions typical for macrocycle synthesis exclusively yielded 2,6-diiminophenylpyridine, whereas unreacted IDA remained in solution (Figures S110-S111). Collectively, these results indicate that pyridine-containing macrocycles have two factors that favor their formation relative to benzene-containing derivatives. First, DFP forms imines more rapidly than IDA. Second, pyridine-containing macrocycles undergo supramolecular polymerization as they form, which further drives macrocycle formation over acyclic products. The corresponding process for IDA macrocycles requires $10^{3}$ higher acid concentrations, such that only more weakly bound assemblies are present during their synthesis.

The most surprising finding of a scrambling experiment was that MC 1 was not formed in detectable amounts, despite there being sufficient DAPB to form a 1:1 mixture of MC 1 and MC 2. This finding suggests that the formation and/or assembly of MC 2 interrupts the templation process required to form MC 1. A scrambling reaction between DAPB ( 1 equiv), DFP ( 0.5 equiv), and IDA ( 0.5 equiv) resulted in the selective formation of MC 2 and small IDA-containing oligomers (Figure 3A). In contrast, both pyridine-2,6-diimine and benzene-1,3-diimine species are formed in the presence of aniline under the same conditions (See Supporting Information). The GPC trace of the scrambling reaction indicated the presence of macrocyclic and oligomeric species. MALDI-MS of the reaction indicated that only MC 2 was formed, and that all identifiable peaks corresponding to oligomers were IDA-containing species (Figures 3B-3C and S60-S66). Furthermore, ${ }^{1} \mathrm{H}$ NMR spectroscopy of the reaction mixture showed resonances corresponding to MC 2, but not MC 1, as well as oligomers containing IDA (Figure S67). Isolation of MC 2 by precipitation into $\mathrm{CH}_{2} \mathrm{Cl}_{2}$ resulted in an isolated macrocycle yield of $72-94 \%$ with respect to DFP, corresponding to half of the available DAPB reacting to yield macrocycles (Table S3). Similar to the direct synthesis of MC 2 and the previous competition experiment, AFM, SEM, and TEM images confirmed the formation of nanotubes that drive macrocycle formation (Figures S68-S70). Lastly, the in situ XRD pattern of the scrambling reaction demonstrates an extended structure analogous to the direct synthesis of MC 2 (Figure S71). These combined findings indicate that the scrambling experiment, conducted at $25 \mathrm{mM}$ DAPB and $12.5 \mathrm{mM}$ each of DFP and IDA, forms MC 2 with no IDA incorporation, as well as no evidence MC 1 formation. In contrast, when $12.5 \mathrm{mM}$ of DAPB and $12.5 \mathrm{mM}$ of IDA are reacted in the absence of DFP, MC 1 is formed in high yield. This suppression of MC 1 formation in the presence of MC 2 was also observed at four other starting concentrations $(12.5 \mathrm{mM}, 8.5 \mathrm{mM}, 6.40 \mathrm{mM}$, and $5.10 \mathrm{mM}$ with respect to DAPB) of the scrambling experiment (See Supporting Information). The results of the scrambling experiment, combined with small molecule experiments and concentration dependent controls, suggest that MC 2 nanotubes disrupt formation of MC 1. 
MC 1 formation was also inhibited in the presence of a pure sample of MC 2, further validating that MC 2 nanotubes prevent the self-templation necessary for MC 1 formation. Furthermore, MC $\mathbf{2}$ was stable to the reaction conditions, despite the presence of free aldehydes, amines, and acid catalyst. Independently synthesized MC 2 nanotubes were added to a solution of DAPB (1 equiv), IDA ( 1 equiv), and $\mathrm{CF}_{3} \mathrm{CO}_{2} \mathrm{H}$ (10 equiv) and left undisturbed for 3 days (Figure 4A). Analysis of the reaction by GPC yielded results analogous to the scrambling reaction in which several elution bands were observed corresponding to discrete macrocycles and imine-linked oligomers (Figure 4B). Subsequent analysis by MALDI-MS demonstrated that MC 2 was retained throughout the process by the lack of oligomers containing DFP moieties as well as no evidence of IDA moieties exchanging into MC 2. The MALDI-MS spectrum contained signals corresponding to IDA-containing oligomers with no DFP incorporation, consistent with the results of the scrambling reactions (Figure 4C). These findings demonstrate that either free MC 2 or nanotubes comprised of MC 2 disrupt the formation of MC 1. Although the self-templation of MC 1 is not understood at the molecular level, these experiments and previous studies point to a templation process driving macrocycle formation, which is disrupted upon inclusion of monomeric or assembled MC 2 species (Figure 2B).

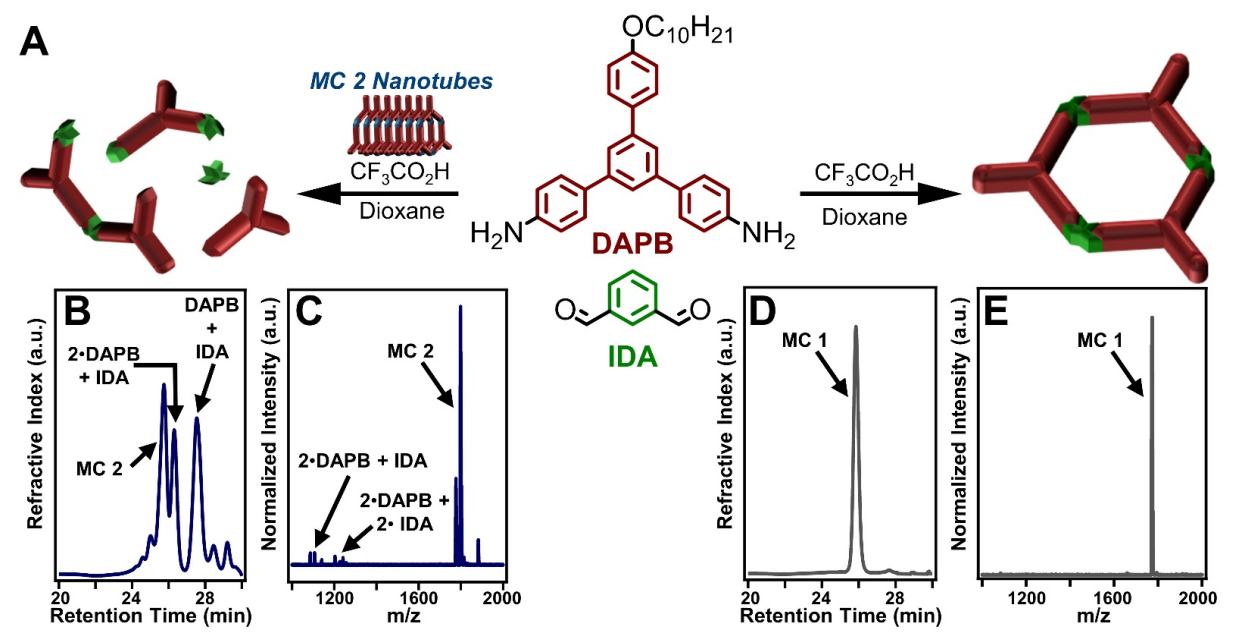

Figure 4. Control experiment demonstrating the effects of MC 2 nanotubes on the selective synthesis of MC 1. (A) Scheme depicting the control experiment in which previously synthesized MC 2 nanotubes are combined with DAPB, IDA, and $\mathrm{CF}_{3} \mathrm{CO}_{2} \mathrm{H}$ under conditions which in the absence of MC 2 nanotubes selectively yields MC 1. (B) Gel permeation chromatogram of the inhibited MC 1 synthesis. (C) MALDI-MS spectra of the results of the inhibited MC 1 synthesis depicting the recovery of MC 2, with no hydrolysis artifacts, and oligomers containing IDA moieties. MC 2 was observed as the $[\mathrm{M}+\mathrm{H}]^{+},[\mathrm{M}+\mathrm{Na}]^{+}$, and $[\mathrm{M}+\mathrm{K}]^{+}$adducts.(D) Representative gel permeation chromatogram of the direct synthesis of MC 1. (E) Representative MALDI-MS spectra of the direct synthesis of MC 1.

Monomer exchange experiments demonstrate that imines within acid-mediated nanotube assembles are far less dynamic than those in weak assemblies or monomeric species. Due to the stability of the imine linkages of MC 2, through a combination of supramolecular polymerization and the inherent chemistry of pyridine2,6-diimine moieties, attempts to exchange its DFP moieties for IDA moieties failed. A previously synthesized sample of MC 2 was resuspended in 1,4-dioxane with IDA (10 equiv), and excess $\mathrm{CF}_{3} \mathrm{CO}_{2} \mathrm{H}$ (Scheme S6). The reaction mixture was sonicated and held at room temperature for 3 days (Figure 5A). Analysis of the monomer exchange product by GPC confirmed the formation of discrete macrocycles (Figure S88). However, analysis of the solution by MALDI-MS showed full recovery of MC 2, with no incorporation of IDA (Figure 5D). ${ }^{1} \mathrm{H}$ NMR spectroscopy of product of the attempted monomer exchange demonstrates recovery of MC 2 with no resonances corresponding to IDA-containing species (Figure 5C). Based on the lack of monomer exchange as demonstrated by MALDI-MS and ${ }^{1} \mathrm{H}$ NMR, we hypothesized that upon exposure to $\mathrm{CF}_{3} \mathrm{CO}_{2} \mathrm{H}, \mathbf{M C} 2$ assembled into nanotubes which, along with the inherent stability 
of pyridine-2,6-diimines, prevented reaction of the imine linkages. This hypothesis was supported by AFM, SEM, and TEM, which depicted the formation of nanotubes akin to the direct synthesis of MC 2 (Figures S92-S94). Lastly, the in situ XRD pattern of the failed monomer exchange matches well with that of the direct MC 2 synthesis (Figure S95). Collectively, the inability to exchange the DFP moieties out of nanotubes assembled from MC 2 highlights the chemical persistence and kinetic stability of the imine linkages within the protonation driven molecular assembly. ${ }^{32}$

Although IDA does not exchange into MC 2 nanotubes, it readily exchanges into macrocycles linked by other substituted isophthalaldehydes. Macrocycles were synthetized using a 5-bromoisophthalaldehyde monomer, which assemble similar to MC 1 (Scheme S8) (Figures S106-S107). Monomer exchange of the 5-bromoisophthalaldehyde linked macrocycles with IDA resulted in the formation of macrocyclic species, as evident by the narrow elution band in the corresponding GPC trace (Scheme S9) (Figure S108). MALDIMS depicts scrambling of the linkages, such that peaks were observed corresponding to macrocycles containing 0,1, 2, or 3 5-bromoisophahlaldehyde moieties (Figure S109). These observations indicate that IDA can exchange into macrocycles that contain similar linkages. IDA's inability to exchange into MC 2 under similar conditions arises either from the increased stability of the pyridine-2,6-diimine moiety or the kinetic persistence of macrocycles in acid-mediated assemblies.
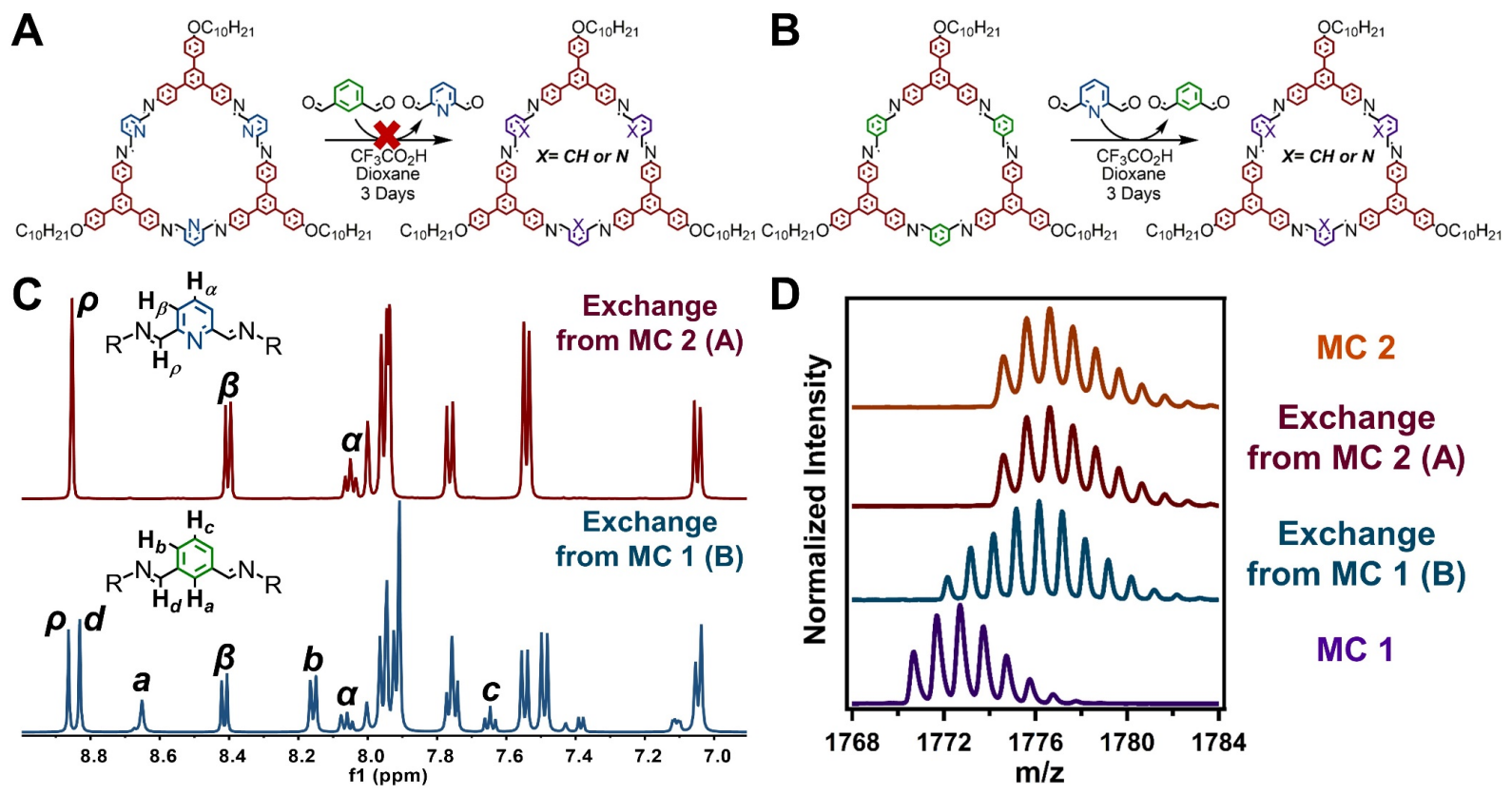

MC 2

Exchange

from MC 2 (A)

Exchange

from MC 1 (B)

MC 1

Figure 5. Monomer exchange of imine-linked macrocycles. (A) Monomer exchange of MC 2 with IDA. (B) Monomer exchange of MC 1 with DFP. (C) ${ }^{1} \mathrm{H}$ NMR spectra of macrocycles resulting from each monomer exchange. (D) MALDI-MS comparison of the macrocycles resulting from each monomer exchange.

When the previous exchange experiment was run in reverse, DFP was able to exchange into MC 1 (Figure $5 \mathrm{~B})$. However, the major product of the exchange is a macrocycle containing only one pyridine-2,6-diimine moiety, despite DFP being used in 10-fold excess with respect to MC 1. MALDI-MS of the products revealed the presence of macrocycles containing a mixture of IDA and DFP subunits (Figure 5D). The spectrum clearly shows no evidence of remaining MC 1 or the fully exchanged MC 2. However, the relative amounts of singly and doubly exchanged macrocycles are not clear. ${ }^{1} \mathrm{H}$ NMR spectroscopy of the reaction indicated that $86 \%$ of the macrocycles contained a single pyridine moiety and $14 \%$ contained two pyridine moieties (Figures 5C and S101). Given the rapid and complete formation of MC 2 from DAPB and DFP, 
even in the presence of IDA, coupled with the thermodynamic preference for pyridine-2,6-diimine linkages; the selective formation of singly and doubly exchanged macrocycles under these conditions strongly suggest that incorporation of a single pyridine unit drives nanotube formation in the presence of $\mathrm{CF}_{3} \mathrm{CO}_{2} \mathrm{H}$ and results in a kinetic trap en route to the thermodynamically favored MC 2 . Indeed, nanotubes were observed in these exchange experiments by AFM, SEM, and TEM (Figures S102-S104). The results of the three monomer exchange experiments highlight the diminished reactivity of imine linkages within acidmediated assemblies. Furthermore, supramolecular polymerization into nanotubes served as a kinetic trap in the full conversion of MC 1 to MC 2, which allows access to non-symmetric macrocycles.

\title{
Conclusions
}

In conclusion, we have developed a system in which supramolecular polymerization is coupled to dynamic covalent bond-forming processes in the synthesis of imine-linked macrocycles. We have demonstrated that the formation of MC $\mathbf{2}$ is kinetically favored relative to MC 1, and its imine linkages are stabilized as a function of acid-mediated supramolecular polymerization and the inherent chemistry of the linkage itself. These three factors led to the selective synthesis of MC 2 dominating a competition experiment with MC 1. Additionally, the mere presence of nanotubes assembled from MC 2 proved to interrupt the synthesis of MC 1, presumably by disrupting the self-templation that guides its selective synthesis. Lastly, monomer exchange experiments demonstrated that once a pyridine-containing dialdehyde exchanged into MC 1 macrocycle, the macrocycle became capable of nanotube formation, which dramatically slowed further imine exchange, and resulted in the kinetic trapping of chemically diverse macrocycles not attainable by direct synthetic methods. These findings highlight the complex interplay of covalent and non-covalent synthesis that can give rise to complex dynamic reaction networks and stimuli responsive materials.

\section{ASSOCIATED CONTENT}

Supporting Information. The Supporting Information is available free of charge on the ChemRxiv Preprint Server:

Experimental procedures and additional characterization data (PDF)

\author{
AUTHOR INFORMATION \\ Corresponding Authors \\ WRD:wdichtel@northwestern.edu
}

\section{ORCID}

Michael J. Strauss: 0000-0003-0808-2568

Austin M. Evans: 0000-0002-3597-2454

Ioannina Castano: 0000-0001-6491-2170

Rebecca Li: 0000-0001-7208-3413

William R. Dichtel: 0000-0002-3635-6119

\section{Notes}

The authors declare no competing financial interests.

\section{Acknowledgement}

This work was funded by the Army Research Office through the Multidisciplinary University Research Initiative (MURI; W911NF-15-1-04477, to W.R.D.). M.J.S was supported by the National Science Foundation (NSF) through the Graduate Research Fellowship Program (GRFP) under Grant No. (DGE1842165). A.M.E. was supported by the NSF through the GRFP under Grant No. (DGE-1324585). I.C. was supported by the NSF through the GRFP under Grant No. (DGE-1842165). I.C. is partially supported by the Ryan Fellowship and the International Institute for Nanotechnology. This work made use of the 
Integrated Molecular Structure Education and Research Center (IMSERC) at Northwestern University, which has received support from the NSF (CHE-1048773), the Soft and Hybrid Nanotechnology Experimental (SHyNE) Resource (NSF; NNCI-1542205), the State of Illinois, and the International Institute for Nanotechnology (IIN). This work also made use of the Scanned Probe Imaging and Development (SPID), and the Electron Probe Instrumentation Center (EPIC), facilities of Northwestern University's Atomic and Nanoscale Characterization Experiment Center (NUANCE), which has received support from the Soft and Hybrid Nanotechnology Experimental (SHyNE) Resource (NSF; ECCS1542205); the MRSEC program (NSF; DMR-1720139) at the Materials Research Center; the International Institute for Nanotechnology (IIN); the Keck Foundation; and the State of Illinois. This work was also supported by the Northwestern University Keck Biophysics Facility and a Cancer Center Support Grant (NCI CA060553). Parts of this work were performed at the DuPont-Northwestern-Dow Collaborative Access Team (DND-CAT) located at Sector 5 of the Advanced Photon Source (APS) at Argonne National Lab. This research used resources of the Advanced Photon Source and the Center for Nanoscale Materials, both U.S. Department of Energy (DOE) Office of Science User Facilities operated for the DOE Office of Science by Argonne National Laboratory under Grant No. (DGE-1324585). We acknowledge Prof. Julia Kalow for the use of her GPC instrument. We acknowledge Prof. Doug Philp for helpful discussions in the preparation of this manuscript.

\section{References}

1. Aida, T.; Meijer, E. W.; Stupp, S. I., Functional Supramolecular Polymers. Science 2012, 335 (6070), 813-817.

2. Würthner, F.; Saha-Möller, C. R.; Fimmel, B.; Ogi, S.; Leowanawat, P.; Schmidt, D., Perylene Bisimide Dye Assemblies as Archetype Functional Supramolecular Materials. Chem. Rev. 2016, 116 (3), 962-1052.

3. Bakker, M. H.; Lee, C. C.; Meijer, E. W.; Dankers, P. Y. W.; Albertazzi, L., Multicomponent Supramolecular Polymers as a Modular Platform for Intracellular Delivery. ACS Nano 2016, 10 (2), 18451852.

4. $\quad$ López-Andarias, J.; Rodriguez, M. J.; Atienza, C.; López, J. L.; Mikie, T.; Casado, S.; Seki, S.; Carrascosa, J. L.; Martín, N., Highly Ordered n/p-Co-assembled Materials with Remarkable Charge Mobilities. J. Am. Chem. Soc. 2015, 137 (2), 893-897.

5. $\quad$ Adelizzi, B.; Aloi, A.; Markvoort, A. J.; Ten Eikelder, H. M. M.; Voets, I. K.; Palmans, A. R. A.; Meijer, E. W., Supramolecular Block Copolymers under Thermodynamic Control. J. Am. Chem. Soc. 2018, 140 (23), 7168-7175.

6. Smulders, M. M. J.; Schenning, A. P. H. J.; Meijer, E. W., Insight into the Mechanisms of Cooperative Self-Assembly: The "Sergeants-and-Soldiers" Principle of Chiral and Achiral C3Symmetrical Discotic Triamides. J. Am. Chem. Soc. 2008, 130 (2), 606-611.

7. Prins, L. J.; Timmerman, P.; Reinhoudt, D. N., Amplification of Chirality: The "Sergeants and Soldiers" Principle Applied to Dynamic Hydrogen-Bonded Assemblies. J. Am. Chem. Soc. 2001, 123 (42), 10153-10163.

8. Helmich, F.; Lee, C. C.; Schenning, A. P. H. J.; Meijer, E. W., Chiral Memory via Chiral Amplification and Selective Depolymerization of Porphyrin Aggregates. J. Am. Chem. Soc. 2010, 132 (47), 16753-16755.

9. Hosono, N.; Palmans, A. R. A.; Meijer, E. W., "Soldier-Sergeant-Soldier" triblock copolymers: revealing the folded structure of single-chain polymeric nanoparticles. Chem. Comm. 2014, 50 (59), 79907993.

10. Mukhopadhyay, R. D.; Ajayaghosh, A., Living supramolecular polymerization. Science 2015, 349 (6245), 241-242.

11. Kang, J.; Miyajima, D.; Mori, T.; Inoue, Y.; Itoh, Y.; Aida, T., A rational strategy for the realization of chain-growth supramolecular polymerization. Science 2015, 347 (6222), 646-651. 
12. Ogi, S.; Sugiyasu, K.; Manna, S.; Samitsu, S.; Takeuchi, M., Living supramolecular polymerization realized through a biomimetic approach. Nat. Chem. 2014, 6, 188.

13. Ogi, S.; Stepanenko, V.; Sugiyasu, K.; Takeuchi, M.; Würthner, F., Mechanism of Self-Assembly Process and Seeded Supramolecular Polymerization of Perylene Bisimide Organogelator. J. Am. Chem. Soc. 2015, 137 (9), 3300-3307.

14. Aliprandi, A.; Mauro, M.; De Cola, L., Controlling and imaging biomimetic self-assembly. Nat. Chem. 2015, 8,10 .

15. Zhang, W.; Jin, W.; Fukushima, T.; Saeki, A.; Seki, S.; Aida, T., Supramolecular Linear Heterojunction Composed of Graphite-Like Semiconducting Nanotubular Segments. Science 2011, 334 (6054), 340-343.

16. Görl, D.; Zhang, X.; Stepanenko, V.; Würthner, F., Supramolecular block copolymers by kinetically controlled co-self-assembly of planar and core-twisted perylene bisimides. Nat. Comm. 2015, 6, 7009.

17. Rupar, P. A.; Chabanne, L.; Winnik, M. A.; Manners, I., Non-Centrosymmetric Cylindrical Micelles by Unidirectional Growth. Science 2012, 337 (6094), 559-562.

18. Qiu, H.; Hudson, Z. M.; Winnik, M. A.; Manners, I., Multidimensional hierarchical self-assembly of amphiphilic cylindrical block comicelles. Science 2015, 347 (6228), 1329-1332.

19. Lin, Q.; Gong, G.-F.; Fan, Y.-Q.; Chen, Y.-Y.; Wang, J.; Guan, X.-W.; Liu, J.; Zhang, Y.-M.; Yao, H.; Wei, T.-B., Anion induced supramolecular polymerization: a novel approach for the ultrasensitive detection and separation of $\mathrm{F}^{-}$. Chem. Comm. 2019, 55 (22), 3247-3250.

20. Venkata Rao, K.; Miyajima, D.; Nihonyanagi, A.; Aida, T., Thermally bisignate supramolecular polymerization. Nat. Chem. 2017, 9, 1133.

21. Kulkarni, C.; Korevaar, P. A.; Bejagam, K. K.; Palmans, A. R. A.; Meijer, E. W.; George, S. J., Solvent Clathrate Driven Dynamic Stereomutation of a Supramolecular Polymer with Molecular Pockets. J. Am. Chem. Soc. 2017, 139 (39), 13867-13875.

22. Johnson, R. S.; Yamazaki, T.; Kovalenko, A.; Fenniri, H., Molecular Basis for Water-Promoted Supramolecular Chirality Inversion in Helical Rosette Nanotubes. J. Am. Chem. Soc. 2007, 129 (17), 57355743.

23. Liu, Y.; Jia, Y.; Zhu, E.; Liu, L.; Qiao, Y.; Che, G.; Yin, B., Supramolecular helical nanofibers formed by an achiral monopyrrolotetrathiafulvalene derivative: water-triggered gelation and chiral evolution. New J. Chem. 2017, 41 (19), 11060-11068.

24. Korevaar, P. A.; de Greef, T. F. A.; Meijer, E. W., Pathway Complexity in $\pi$-Conjugated Materials. Chem. Mater. 2014, 26 (1), 576-586.

25. van der Zwaag, D.; Pieters, P. A.; Korevaar, P. A.; Markvoort, A. J.; Spiering, A. J. H.; de Greef, T. F. A.; Meijer, E. W., Kinetic Analysis as a Tool to Distinguish Pathway Complexity in Molecular Assembly: An Unexpected Outcome of Structures in Competition. J. Am. Chem. Soc. 2015, 137 (39), 12677-12688.

26. Valera, J. S.; Gómez, R.; Sánchez, L., Supramolecular Polymers: Tunable Energy Landscapes to Control Pathway Complexity in Self-Assembled N-Heterotriangulenes: Living and Seeded Supramolecular Polymerization. Small 2018, 14 (3), 1870012.

27. $\quad$ Engel, S.; Spitzer, D.; Rodrigues, L. L.; Fritz, E.-C.; Straßburger, D.; Schönhoff, M.; Ravoo, B. J.; Besenius, P., Kinetic control in the temperature-dependent sequential growth of surface-confined supramolecular copolymers. Faraday Discuss. 2017, 204 (0), 53-67.

28. Carnall, J. M. A.; Waudby, C. A.; Belenguer, A. M.; Stuart, M. C. A.; Peyralans, J. J.-P.; Otto, S., Mechanosensitive Self-Replication Driven by Self-Organization. Science 2010, 327 (5972), 1502-1506.

29. Nguyen, R.; Allouche, L.; Buhler, E.; Giuseppone, N., Dynamic Combinatorial Evolution within Self-Replicating Supramolecular Assemblies. Angew. Chem. Int. Ed. 2009, 48 (6), 1093-1096.

30. Williams, R. J.; Smith, A. M.; Collins, R.; Hodson, N.; Das, A. K.; Ulijn, R. V., Enzyme-assisted self-assembly under thermodynamic control. Nat. Nanotechnol. 2008, 4, 19. 
31. Strauss, M.J.; Asheghali, D.; Evans, A.M.; Li, R.L.; Chavez, A.D.; Sun, C.; Becker, M. L.; Dichtel, W. R., Cooperative Self-Assembly of Pyridine-2,6-Diimine-Linked Macrocycles into Mechanically Robust Nanotubes. Angew. Chem. Int. Ed. 10.1002/anie.201907668.

32. Sun, C.; Shen, M.; Chavez, A. D.; Evans, A. M.; Liu, X.; Harutyunyan, B .; Flanders, N. C.; Hersam, M. C.; Bedzyk, M. J.; Olvera de la Cruz, M.; Dichtel, W. R., High aspect ratio nanotubes assembled from macrocyclic iminium salts. Proc. Natl. Acad. Sci. U.S.A. 2018, 115 (36), 8883-8888.

33. Chavez, A. D.; Evans, A. M.; Flanders, N. C.; Bisbey, R. P.; Vitaku, E.; Chen, L. X.; Dichtel, W. R., Equilibration of Imine-Linked Polymers to Hexagonal Macrocycles Driven by Self-Assembly. Chem. Eur. J. 2018, 24 (16), 3989-3993.

34. Giuseppone, N.; Lehn, J.-M., Protonic and Temperature Modulation of Constituent Expression by Component Selection in a Dynamic Combinatorial Library of Imines. Chem. Eur. J. 2006, 12 (6), 17151722.

35. Godoy-Alcántar, C.; Yatsimirsky, A. K.; Lehn, J.-M., Structure-stability correlations for imine formation in aqueous solution. J. Phys. Org. Chem. 2005, 18 (10), 979-985.

36. Kulchat, S.; Chaur, M. N.; Lehn, J.-M., Kinetic Selectivity and Thermodynamic Features of Competitive Imine Formation in Dynamic Covalent Chemistry. Chem. Eur. J. 2017, 23 (46), 11108-11118. 37. Schultz, D.; Nitschke, J. R., Kinetic and Thermodynamic Selectivity in Subcomponent Substitution. Chem. Eur. J. 2007, 13 (13), 3660-3665. 